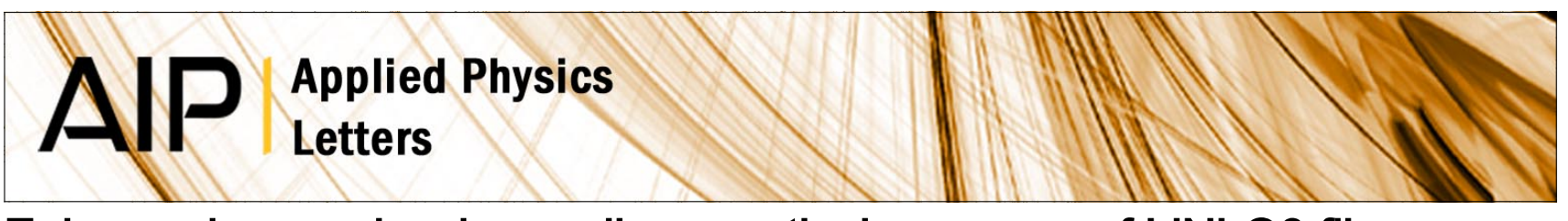

\title{
Enhanced second-order nonlinear optical response of LiNbO3 films upon Er doping
}

\author{
J. Gonzalo, J. A. Chaos, A. Suárez-García, C. N. Afonso, and V. Pruneri
}

Citation: Appl. Phys. Lett. 81, 2532 (2002); doi: 10.1063/1.1506949

View online: http://dx.doi.org/10.1063/1.1506949

View Table of Contents: http://apl.aip.org/resource/1/APPLAB/v81/i14

Published by the American Institute of Physics.

\section{Related Articles}

Analyzing photo-induced interfacial charging in IZO/pentacene/C60/bathocuproine/Al organic solar cells by electric-field-induced optical second-harmonic generation measurement

J. Appl. Phys. 111, 113711 (2012)

Cherenkov high-order harmonic generation by multistep cascading in $\mathrm{X}(2)$ nonlinear photonic crystal Appl. Phys. Lett. 100, 221103 (2012)

Multielectron effects in high harmonic generation in N2 and benzene: Simulation using a non-adiabatic quantum molecular dynamics approach for laser-molecule interactions

J. Chem. Phys. 136, 194303 (2012)

First-principle description for the high-harmonic generation in a diamond by intense short laser pulse J. Appl. Phys. 111, 093112 (2012)

Phase matching for surface plasmon enhanced second harmonic generation in a gold grating slab Appl. Phys. Lett. 100, 181107 (2012)

\section{Additional information on Appl. Phys. Lett.}

Journal Homepage: http://apl.aip.org/

Journal Information: http://apl.aip.org/about/about_the_journal

Top downloads: http://apl.aip.org/features/most_downloaded

Information for Authors: http://apl.aip.org/authors

\section{ADVERTISEMENT}

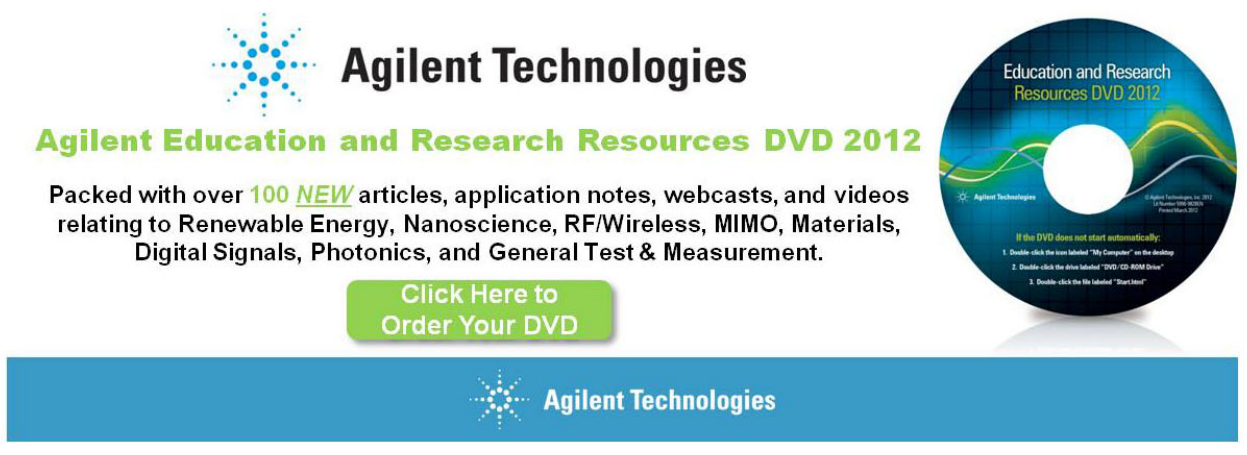




\title{
Enhanced second-order nonlinear optical response of $\mathrm{LiNbO}_{3}$ films upon Er doping
}

\author{
J. Gonzalo, J. A. Chaos, A. Suárez-García, and C. N. Afonso ${ }^{a)}$ \\ Instituto de Optica, C.S.I.C., Serrano 121, 28006 Madrid, Spain \\ V. Prunerib) \\ Optoelectronics Research Centre, University of Southampton, United Kingdom
}

(Received 6 May 2002; accepted for publication 18 July 2002)

\begin{abstract}
Erbium-doped $\mathrm{LiNbO}_{3}$ films have been produced in a single-step process by alternate pulsed laser deposition. The dopant is incorporated in submonolayers whose nominal indepth separation is varied in the range 1.7-4.0 $\mathrm{nm}$ to lead respectively to Er concentrations in the range 3.4-0.6 $\times 10^{20}$ atoms $/ \mathrm{cm}^{-3}$. All the films exhibit the characteristic $\mathrm{Er}^{3+}$ photoluminescence at $1.54 \mu \mathrm{m}$ with lifetime values as high as $3 \mathrm{~ms}$. The $d_{33}$ nonlinear coefficients determined from second-harmonic generation experiments are in the range $22-28 \mathrm{pm} / \mathrm{V}$, the films having nominal Er submonolayer indepth separation of 3-4 nm exhibiting $d_{33}$ values slightly above that of the bulk material. The comparison of the $d_{33}$ values obtained in the Er-doped films to those reported earlier for similar undoped films shows clearly that both the structural quality and the second-harmonic performance of the films can be enhanced by Er doping. (c) 2002 American Institute of Physics. [DOI: $10.1063 / 1.1506949$ ]
\end{abstract}

Single-crystalline $\mathrm{LiNbO}_{3}(\mathrm{LN})$ has a variety of optical properties that make it a key material for the development of many optical devices. A worldwide research effort have been made to incorporate optically active ions such as rare earths for improved gain devices ${ }^{1}$ and to enhance the second-order nonlinear optical response by producing periodically poled LN crystals. ${ }^{2}$ The promising performances foreseen to be achieved by the combination of both functionalities (i.e., amplification and second-order nonlinearity) in the same material has led to an intense work in the production of rare-earth doped LN crystals with an enhanced nonlinear response. ${ }^{3}$ Most of the studies focus either on the ion luminescence performance or on the nonlinear response, with very little discussion on the possible interaction of both responses. Beneficial and detrimental effects have both been reported in LN upon Er doping such as an improvement of the two-color recording performance ${ }^{4}$ and a $4 \%-5 \%$ increased dispersion in the domain period length upon periodically poling, ${ }^{3}$ respectively. Positive interactions have also been reported in other systems such as an increased resistance of $\chi^{2}$ gratings to erasure in rare-earth doped aluminosilicate fibers ${ }^{5}$ or an enhancement of the effective nonlinear coefficient of $\mathrm{BaTiO}_{3}$ upon cerium doping. ${ }^{6}$

The strong research activity in bulk crystals has partially been mirrored in waveguides produced by different techniques including ion exchange techniques, ${ }^{7}$ metal indiffusion, ${ }^{1}$ or ion implantation. ${ }^{8}$ These works have been focused to the study of the $\mathrm{Er}^{3+}$ photoluminescence (PL) performance at $1.54 \mu \mathrm{m}$. No similar activity has been reported in the case of waveguides prepared by thin-film technologies probably because the growth of good quality LN films has for a long time been hindered due to the Li defi-

\footnotetext{
a)Electronic mail: cnafonso@io.cfmac.csic.es

b) Present address: Corning-OTI, Viale Sarca 222, 20126 Milano, Italy.
}

ciency of the films. The use of pulsed laser deposition helped to overcome this problem by producing highly textured $\mathrm{LN}$ films. ${ }^{9-12}$ Whereas there are some reports on the secondorder nonlinear response of $\mathrm{LN}$ textured films, ${ }^{11,12}$ to our knowledge, there is just one work reporting the successful doping of textured LN films by covering the substrate with an Er layer that was later indiffused by annealing thus leading to a nonhomogeneous Er profile. ${ }^{13}$ The aim of this work is twofold: First, to study the influence of doping LN with $\mathrm{Er}$ on the second-harmonic (SH) response, and second, to report the growth of strongly textured Er-LN films in a single-step process, with a controlled depth profile and with excellent PL and SH performances when compared to those of the bulk crystalline material.

Film deposition was performed by focusing an ArF laser beam (pulse duration of $20 \mathrm{~ns}$ ) at $45^{\circ}$ alternately in a LN single crystal and in an Er (99\%) target. The process was repeated a number of times (cycles), alternating one pulse on the Er target with a number of pulses on the LN target. The number of cycles and the number of pulses in the LN target were varied in order to change the Er indepth profile while keeping the total film thickness approximately constant. The substrate-target distance was $\approx 30 \mathrm{~mm}$ and the laser energy density was $1.2 \mathrm{~J} / \mathrm{cm}^{-2}$. The deposition was performed in an oxygen pressure of 1 Torr on (001) sapphire substrates held at $650^{\circ} \mathrm{C}$, these conditions being those earlier reported as optimum for the production of undoped LN films. ${ }^{12}$ After deposition, all films were cooled down in situ in an oxygen pressure of 75 Torr. The films were finally annealed in air at $700{ }^{\circ} \mathrm{C}$ during $1 \mathrm{~h}$ prior to any measurement.

The film thickness and the Er content were determined by Rutherford backscattering spectrometry (RBS) using a 2 $\mathrm{MeV} \mathrm{He}^{+}$beam and a scattering angle of $150^{\circ}$. The thickness was calculated from the measured $\mathrm{Nb}$ areal density using the density of bulk LN. The Er content could only be 
TABLE I. Parameters used the growth of Er-doped LN films and data obtained from RBS measurements (average Er concentration in atoms $/ \mathrm{cm}^{-3}$, film thickness, and nominal Er submonolayers indepth separation).

\begin{tabular}{ccccc}
\hline $\begin{array}{c}\text { No. of } \\
\text { cycles }\end{array}$ & $\begin{array}{c}\text { No. of pulses on } \\
\text { LN per cycle }\end{array}$ & $\begin{array}{c}\text { Er } \\
10^{20} \text { atoms/cm }\end{array}$ & $\begin{array}{c}\text { thickness } \\
(\mathrm{nm})\end{array}$ & $\begin{array}{c}\text { Er separation } \\
(\mathrm{nm})\end{array}$ \\
\hline 150 & 1380 & 3.4 & $254 \pm 5$ & 1.7 \\
85 & 2730 & 2.0 & $243 \pm 5$ & 2.9 \\
55 & 4740 & 0.65 & $222 \pm 5$ & 4.0 \\
\hline \hline
\end{tabular}

experimentally determined in the case of the film having the highest Er content (150 cycles) and resulted to be $5 \pm 3$ $\times 10^{13}$ atoms $/ \mathrm{cm}^{-2}$ per layer that is less than a monolayer. ${ }^{14}$ This was due to the fact that the $\mathrm{Er}$ and $\mathrm{Nb}$ signals appear very close in the RBS spectra combined with the low areal density of Er. From the Er content determined for this film, we calculated the number of Er atoms per layer and we assumed the same value in all the films. By multiplying this value for the number of cycles, the content of Er was determined for the films made with 85 and 55 cycles and the average Er concentration in the film could be estimated from these data. The nominal separation between the Er submonolayers was calculated by dividing the thickness of the film by the number of cycles. The results are all included in Table I where it is seen that the Er average concentration decreases as the nominal submonolayer separation increases because the films were designed to have a similar total thickness. In spite of this and the small thicknesses $(222-254 \mathrm{~nm})$, it is clearly seen that all films have an Er average content close or above $10^{20}$ atoms $/ \mathrm{cm}^{-3}$, that is considered the minimum concentration required to achieve amplification. ${ }^{15}$

The structure of the films was analyzed by normal incidence $\theta-2 \theta$ and $\phi$ x-ray diffraction (XRD). The films become textured as the Er submonolayer indepth separation increased: Whereas no peaks related to $\mathrm{LN}$ could be observed in the film having $1.7 \mathrm{~nm}$ separation, a sharp peak from the (006) reflection of LN is the only one appearing in the $\theta-2 \theta$ scan of the film having the largest separation as seen in Fig. 1 (a). This scan is very similar to that reported earlier for the undoped films as seen in Fig. 1(b). ${ }^{12}$ The crystalline quality of the films was earlier evaluated through the evolution of the full width half maximum (FWHM) of the (006) reflection of LN. Using as a normalization factor, the width of the peak corresponding to the sapphire substrate $\left(0.2^{\circ}\right)$, the FWHM values calculated from the scans in Fig. 1 are 1.0 and 1.4 for the doped and undoped fims, respectively. Provided that the doped film is half as thick than the undoped one and that it was earlier reported that the quality of the undoped films increased as the thickness was increased, ${ }^{12}$ it can be concluded that the quality of the films improve by $\mathrm{Er}$ doping with an Er submonolayer separation of $4 \mathrm{~nm}$.

The insets in Fig. 1 show to the $\phi$-scan patterns. They exhibit peaks when the films are rotated by $60^{\circ}$ around the normal axis that indicates the existence of two structural variants (twined crystals). The peak intensity ratio of these two variants is slightly higher (2.4 compared to 2.0) for the doped film, thus indicating smaller twinning. Since the variant showing the more intense peaks is the one aligned with the substrate, it can be deduced that the epitaxial growth is favored by the Er doping in the film having an Er submono-

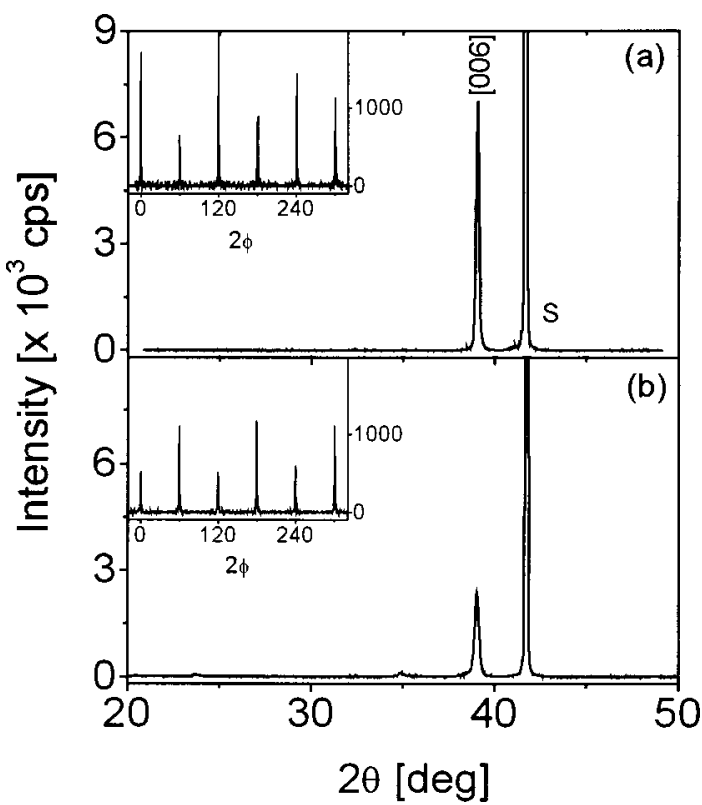

FIG. 1. $\theta-2 \theta$ scans of: (a) a $222 \mathrm{~nm}$ thick LN film doped with Er submonolayers having a nominal indepth separation of $4.0 \mathrm{~nm}$ and (b) a $500 \mathrm{~nm}$ thick LN undoped film (taken from Ref. 12). The peaks labeled (006) and S correspond to $\mathrm{LN}$ and the sapphire substrate, respectively. The insets show the corresponding $\phi$ scans.

layer indepth separation of $4 \mathrm{~nm}$. It can be concluded that the epitaxial growth of LN on sapphire substrates is favored upon doping with Er submonolayers separated $4 \mathrm{~nm}$.

The PL of $\mathrm{Er}^{3+}$ at $1.54 \mu \mathrm{m}$ was excited at room temperature by using an $\mathrm{Ar}^{+}$ion laser tuned to its $514.5 \mathrm{~nm}$ line and focused into the specimen at $25^{\circ}$ with its normal direction. The pump power was $150 \mathrm{~mW}$ and the PL was collected along the normal direction and detected by a liquid-nitrogencooled Ge detector. Further details can be found elsewhere. ${ }^{14}$ Figure 2 (a) shows that the PL intensity decreases as the Er submonolayer separation increases, that is consistent with the decrease in the total number of $\mathrm{Er}^{3+}$ ions indicated as the top axis. This behavior is in contrast to that of the PL lifetime that is seen to increase up to $3 \mathrm{~ms}$ as the Er submonolayer indepth separation increases. In an earlier work, the same procedure of doping with $\mathrm{Er}$ submonolayers was reported for the case of an $\mathrm{Al}_{2} \mathrm{O}_{3}$ host. ${ }^{16}$ The range of separations studied was broader $(0.6-9 \mathrm{~nm})$ and it was shown that the lifetime was greatly increased for separations above $3 \mathrm{~nm}$, and the increase was saturated for distances in the range 4-6 nm. The good agreement of the present results with those reported earlier in Ref. 16 suggests that a separation in the range 3-6 $\mathrm{nm}$ between Er submonolayers is required to prevent concentration quenching effects. It should be pointed out that although the distances empirically estimated in these two examples are similar, the real distances might be smaller in the case of the LN. The $\mathrm{Er}_{\mathrm{Al}} \mathrm{Al}_{2} \mathrm{O}_{3}$ films reported elsewhere ${ }^{14,16}$ were deposited at room temperature and step annealed to $850^{\circ} \mathrm{C}$ whereas the $\mathrm{LN}$ films in this work were deposited at $650{ }^{\circ} \mathrm{C}$ and annealed at $700^{\circ} \mathrm{C}$. The different growth temperature might result in larger diffusion lengths during growth in the case of LN and thus, the real separations might be smaller than those calculated. This reasoning supports further the previous conclusion related to the need for a minimum Er submonolayer indepth separation to pre- 


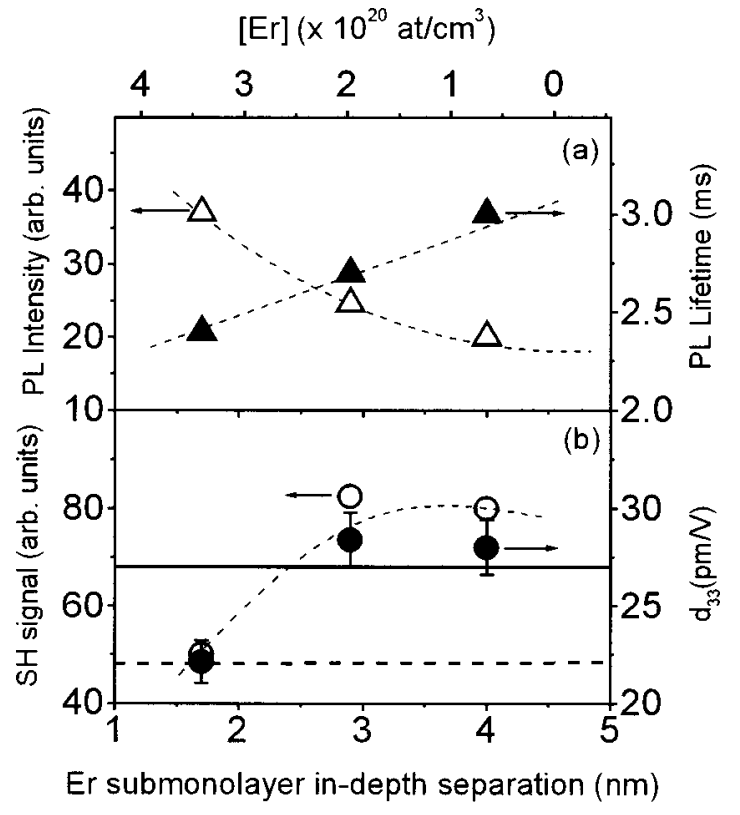

FIG. 2. (a) PL intensity and lifetime at $1.54 \mu \mathrm{m}$ and (b) SH signal and effective nonlinear coefficient $\left(d_{33}\right)$ as a function of the Er submonolayer indepth separation in Er-doped LN films. The average Er concentration is quoted for reference at the the top axis. The dashed lines connecting the experimental points are guides for the eyes whereas the horizontal lines in (b) mark the $d_{33}$ value for (solid) the bulk single-domain crystal (taken from Ref. 17) and (dashed) a $500 \mathrm{~nm}$ thick undoped film (taken from Ref. 12).

vent concentration quenching effects and suggests that lifetime values well above $3 \mathrm{~ms}$ should be easily achievable in Er-doped LN films if using nominal separations larger than 4 $\mathrm{nm}$. It is worth noting that the highest lifetime achieved (3 $\mathrm{ms}$ ) is already slightly higher than the lifetime values reported in waveguides produced by ion implantation having an Er concentration close to the quenching limit ${ }^{8}$ or much higher than those reported for ion-exchange waveguides, ${ }^{7}$ the latter having a very inhomogeneous Er indepth profile together with Li deficiencies.

The second-order nonlinear optical properties of the annealed films were measured via second-harmonic generation using a $Q$-switched and mode-locked Nd:YAG laser operating at $1.064 \mu \mathrm{m}$. The experimental setup used and the procedure followed to determine $d_{33}$ involved the use a quartz sample as a reference leading to the determination of $d_{33}$ within $\pm 5 \%$. The details have been described elsewhere. ${ }^{12}$ Figure 2 (b) shows that the SH signal and the $d_{33}$ coefficient both increase as the Er submonolayer indepth separation increases from 1.7 to $2.9 \mathrm{~nm}$ and undergoes a maximum or reaches a nearly constant value for separations in the range 2.9-4.0 nm. Two horizontal lines are plotted in Fig. 2 (b) that relate to the value reported for single-domain crystals ${ }^{17}$ and the one reported earlier for a $500 \mathrm{~nm}$ thick undoped LN film grown under the same conditions. ${ }^{12}$ Their comparison shows that the $d_{33}$ values achieved in the doped films are clearly higher than those in the undoped films and that they are close to or slightly higher than those of the bulk material for Er submonolayer separations in the range $2.9-4.0 \mathrm{~nm}$. The origin of this enhancement is not clear at this point and needs more investigation. An increase of 1.43 times of the dielectric function of the doped material in respect to the pure crystal and an increase in the polarization along $c$ caused by an increase in the ratio $c / a$ were reasons given earlier to explain the $d_{33}$ enhancement observed in $\mathrm{BaTiO}_{3}$ films upon doping with $\mathrm{Ce} .{ }^{6}$ Although no measurements of the dielectric function of our LN films are available, it should increase as the Er content increases that is not the behavior observed in $d_{33}$ in our films. Whether or not a change of ratio $c / a$ occurs in our doped LN films can not be deduced from the XRD experiments performed. Nevertheless, this explanation is also unlikely since the film having an Er submonolayer separation of $2.9 \mathrm{~nm}$ exhibits a large enhancement in $d_{33}$ although its $\theta-2 \theta$ scan shows no particular texture.

In conclusion, highly textured $\mathrm{LiNbO}_{3}$ films doped with Er up to concentrations close to $10^{20}$ atoms $/ \mathrm{cm}^{-3}$ have been grown on sapphire substrates by pulsed laser deposition, the crystalline quality of the films being enhanced upon Er doping. Both the structural quality of the films and the $\mathrm{Er}^{3+}$-related PL lifetime at $1.54 \mu \mathrm{m}$ are found to improve more the larger the indepth separation of the Er submonolayers. The $d_{33}$ nonlinear coefficient of the doped films is close to or above that of the bulk single-crystalline material and it is clearly much better than that of thicker undoped films, thus proving that Er doping enhances the SH response.

This work was partially supported by CICYT (Spain) under Project No. TIC99-0866 and the Acción Integrada Hispano-Británica programme (HB1999-0149). The authors thank Dr. D. Hole from University of Sussex (UK) for the RBS measurements, Dr. A. Cebollada from Instituto de Microelectrónica Madrid (CSIC, Spain) for assistance with the XRD analysis and helpful discussions, and Dr. R. Serna from Instituto de Optica (CSIC, Spain) for helpful discussions.

${ }^{1}$ C. Becker, T. Oesselke, J. Pandavenes, R. Ricken, K. Rockhausen, G. Schreiber, W. Sohler, H. Suche, R. Wessel, S. Balcamo, I. Montrosset, and D. Sciancalepore, IEEE J. Sel. Top. Quantum Electron. 6, 101 (2000).

${ }^{2}$ L. E. Myers, R. C. Eckardt, M. M. Fejer, R. L. Byer, W. R. Bosenberg, and J. W. Pierce, J. Opt. Soc. Am. B 12, 2102 (1995).

${ }^{3}$ V. Bermúdez, J. Capmany, J. García Solé, and E. Dieguez, Appl. Phys. Lett. 73, 593 (1998)

${ }^{4}$ Y. Tomita, M. Hoshi, and S. Sunarno, Jpn. J. Appl. Phys., Part 2 40, L1035 (2001).

${ }^{5}$ E. M. Dianov, L. S. Kornienko, V. I. Stupina, and P. V. Chernov, Opt. Lett. 20, 1253 (1995).

${ }^{6}$ L-Z Xuan, S-H Pan, Z-H Chen, R-P Wang, W-S Shi, and C-L Li, Appl. Phys. Lett. 73, 2896 (1998).

${ }^{7}$ C. Sada, E. Borsella, F. Caccavale, F. Gonella, F. Segato, Y. N. Korkishko, V. A. Fedorov, T. M. Morozova, G. Battaglin, and R. Polloni, Appl. Phys. Lett. 72, 3431 (1998).

${ }^{8}$ M. Fleuster, C. Buchal, E. Snoeks, and A. Polman, J. Appl. Phys. 75, 173 (1994).

${ }^{9}$ S. B. Ogale, R. Nawathey-Dikshit, S. J. Dikshit, and S. M. Kanetckar, J. Appl. Phys. 71, 5718 (1992).

${ }^{10}$ X. L. Wu, X. L. Guo, Z. G. Liu, S. S. Jiang, and D. Feng, Appl. Phys. Lett. 69, 3963 (1996)

${ }^{11}$ D-W. Kim, S-H. Lee, and T. W. Noh, Mater. Sci. Eng., B 56, 251 (1998).

${ }^{12}$ J. A. Chaos, V. Pruneri, J. Gonzalo, and C. N. Afonso, J. Appl. Phys. 88, 3768 (2000).

${ }^{13}$ S. Bauer, L. Beckers, M. Fleuster, J. Schubert, W. Zander, and C. Buchal, Mater. Res. Soc. Symp. Proc. 341, 283 (1994).

${ }^{14}$ R. Serna, M. Jiménez de Castro, J. A. Chaos, and C. N. Afonso, Appl. Phys. Lett. 75, 4073 (1999).

${ }^{15}$ A. Polman, J. Appl. Phys. 82, 1 (1997).

${ }^{16}$ R. Serna, M. Jiménez de Castro, J. A. Chaos, A. Suárez-García, C. N. Afonso, M. Fernández, and I. Vickridge, J. Appl. Phys. 90, 5120 (2001).

${ }^{17}$ D. A. Roberts, IEEE J. Quantum Electron. 28, 2057 (1992). 